\title{
Hydrogels; A Novel Drug Delivery System
}

\author{
Tayyaba Rana ${ }^{1}$, Zainab Naeem ${ }^{1}$, Sumiyya Javaid ${ }^{1}$, Nayab Sajid ${ }^{1}$, Aamna Habib ${ }^{1}$, Madeeha Fatima ${ }^{2}$, \\ Muhammad Imran Khan ${ }^{1}$ and Abdul Qayyum Khan ${ }^{3 *}$
}

${ }^{1}$ Pharm-D (2013-2018) MPhil Pharmaceutics, University of central Punjab, Lahore, Pakistan

${ }^{2}$ Lecturer, The University of Faisalabad, Pakistan

${ }^{3}$ Pharm-D, MPhil, PhD, Hannover Medical School, Germany

*Corresponding author: Abdul Qayyum Khan, Pharm-D, MPhil, PhD, Hannover Medical School, Germany

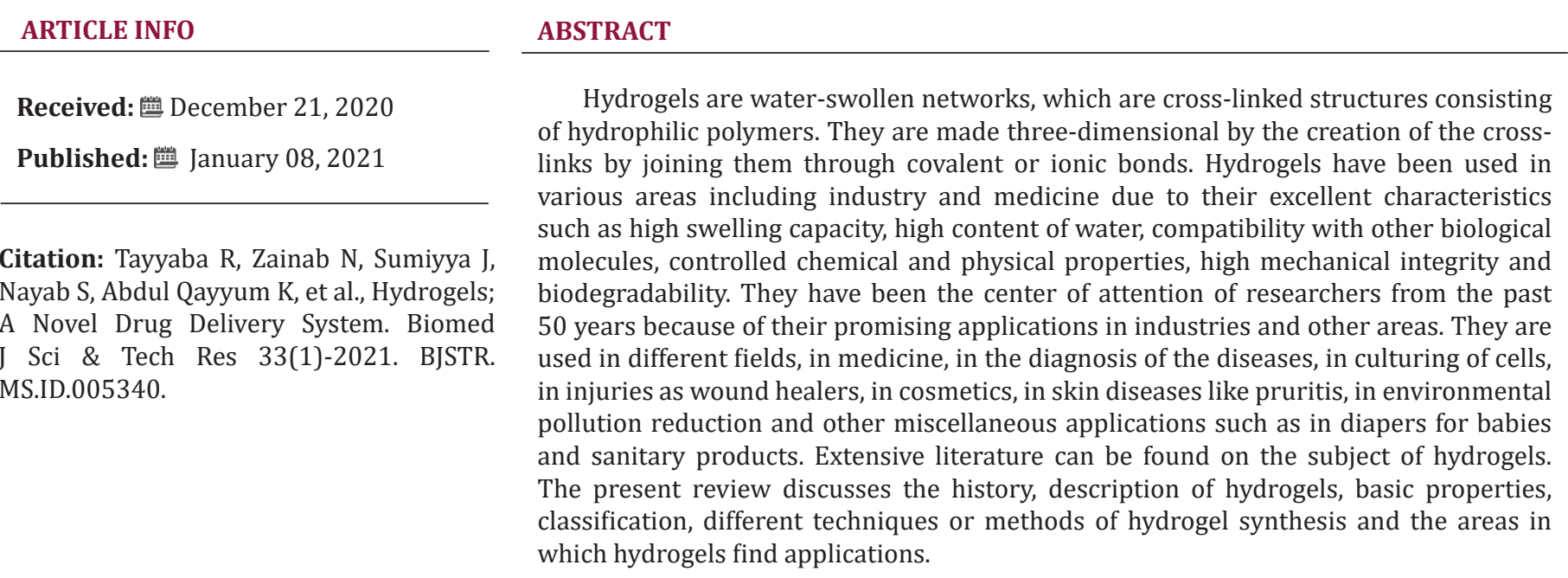

\section{Introduction}

\section{History}

The word "hydrogel", as stated by Lee, Kwon and Park, has been used since 1894 but that material was not a hydrogel but a colloidal gel (of inorganic salts) [1]. These gels are made of polymeric matrices but they do not dissolve instead they swell [2]. Anyhow, the first accurate hydrogel having a cross-linked network was reported by Wichterle and Lim in 1960 for the first time [3]. It was a polyhydroxy ethyl methacrylate hydrogel that was synthesized with the aim of its usage in permanent contacts. Hydrogels are the first to be synthesized for uses inside the patient. After that, the research on the topic of hydrogels and their biomedical applications started to rise [4]. Some influential and important work was performed on hydrogels in the 1980s by Lim and Sun [5].

They have been used in medicine from about fifty years ago. Their history goes way back although the discussion in literature involves more information about their use in medical and pharmaceutical areas. The history of hydrogels has been classified in three different generations. The first generation included crosslinking techniques that involved chemical alterations. These modifications were applied to achieve high swelling and good mechanical characteristics [6]. The second generation contains materials that are sensitive to and respond to specific stimuli, for example, $\mathrm{pH}$, concentration, and temperature. The secondgeneration hydrogels were made to overcome the problems of mechanical strength. Finally, the third generation then focused on investigating and developing stereo complex materials and hydrogels that were cross-linked through physical interactions. This development led to the development of "smart hydrogels" that are polymeric matrices with a broad spectrum of tailorable attributes [7]. These gels maintain their stability in the fluctuating condition such as temperature [8].

\section{What are Hydrogels?}

A hydrogel can be described as a three dimensional network formed by hydrophilic polymers which can expand in water. These polymers can hold copious amounts of water without disrupting 
the structure [9]. Some researchers define it as a swollen polymeric material retaining a significant volume of water in it without itself being dissolved in water [10]. Hydrogels are novel drug deliverers that can aid in the delivery of several kinds of drug molecules either therapeutic or diagnostic nature. They are also suitable carriers for immunological products such as vaccines and other biological products like plasmas and seras and valvular intestinal cells [11]. Hydrogels, owing to their high water load, show flexibility that is similar to natural tissue. They owe this property to the existence of different functional groups in them that are hydrophilic. These groups include:
a) $-\mathrm{COOH}$,
b) $-\mathrm{NH}_{2}$
c) $-\mathrm{OH}$,
d) $-\mathrm{CONH}$
e) $-\mathrm{CONH}_{2}$ and
f) $-\mathrm{SO}_{3} \mathrm{H}[12]$

A polyampholyte hydrogel consists of negative and positive ions are bound to the backbone [13]. The resistance of hydrogels to dissolution occurs due to the cross-linkage between the chains in the network [14]. This network can include both natural and synthetic materials. The synthetic hydrogels are gradually taking the place of the natural ones during the last two decades due to the better properties presented by the synthetic hydrogels for instance, high water absorption capacity and incredible strength. They show a well-defined morphology which can also be modified or altered to get desired traits such as strength, functionality and biodegradability [15]. Hydrogels can undergo transitions such as gel-sol transition or transitions in the volume phase as a result of different biochemical stimuli and physical or chemical stimuli [16]. Physical stimuli comprise electric fields, temperature, pressure, light intensity, the composition of the solvent and magnetic field. In contrast, the chemical and biochemical stimuli involve $\mathrm{pH}$, chemical compositions, and various ions. In most cases, these transitions are reversible. A hydrogel's response to stimuli is dependent on the charge density, nature of monomers, and cross-linkage [17]. The charged hydrogels swell when they are exposed to the electrical field and undergo shape changes [18].

\section{Classification of Hydrogels}

The main constituents of the hydrogels are biopolymers or polyelectrolytes [19]. Hydrogels can be divided into different types according to the source from which they have been and the ionic charges, appearance, configuration and type of cross-linkages. These are classified based on:

\section{Source}

Natural Origin: These hydrogels contain natural polymers, for instance, proteins (gelatin and collagen) and other polysaccharides (starch, agarose, and alginate) [20].

Synthetic Origin: These are constituted from synthetic polymers that are synthesized by chemical polymerization methods [21].

\section{Nature of Hydrogel}

Hydrogels can be of different types in nature [22].

Physical Gels: These transitions from liquid to gel in turn of environmental changes ( $\mathrm{pH}$, temperature or pressure) or mixing. Physical gels are also called as reversible gels.

Chemical Gels: These gels involve covalent bonding for mechanical integrity and resistance to degradation. These gels are also called as permanent gels.

Biochemical Gels: These involve biological agents such as amino acids or enzymes as participants of the gelation process.

\section{Configuration}

Hydrogels can be put into different classes depending on their morphology such as:

a) Amorphous (non-crystalline).

b) Semicrystalline.

c) Crystalline [23].

\section{Physical Appearance}

Hydrogels can be organized into various classes based on how they appear, these are as follows:

a) iMatrix

b) Film

c) Microsphere

This appearance usually depends on the polymerization method being used in the preparation process [24].

\section{Type of Cross-Linking}

The cross-link junctions in the hydrogels can have chemical or physical nature.

a) Chemical cross-linkage has a permanent junction.

b) Physical cross-linkages have transient junctions [25].

\section{Polymeric Composition}

Classification into different types on the method of their preparation is as follows: 
Homopolymeric Hydrogels: Contain a polymeric network having a single monomer species [26]. These hydrogels can be used as scaffolds for the promotion of cell adhesion and the regeneration of spinal cord cells [27].

Copolymeric Hydrogels: Comprise of two or more species of different monomers with a minimum of one lipophobic component. These components are usually arranged in different configurations, such as random or block configuration along the polymer network chain. For example, cellulose and CMC were used in the manufacture of PVP based hydrogels [28].

Semi-Interpenetrating Polymeric Hydrogels: This network is formed by penetration of one linear chained polymer into another cross-linked network and they have no chemical bonds in between them [29].

Interpenetrating Polymeric Hydrogels (IPN): This class has two synthetic or natural independent and cross-linked polymeric components in a network configuration [30].

\section{Electrical Charge}

a) Nonionic - having no charge [31].

b) Ionic - having either a negative or positive charge.

c) Amphoteric electrolyte (ampholytic).

d) Zwitterionic [32].

\section{Properties of Hydrogels}

Hydrogels are finding many applications in domestic and industrial areas due to its properties. Hydrogels have:

a) Both solid and liquid-like properties

b) High biocompatibility [33]

c) Maximum absorption capacity

d) Preferred particle size and desired porosity.

e) Shrink on drying

f) Responsive to stimuli [34].

\section{Swelling}

Hydrogels are polymers with cross-linkages that become swollen in a liquid medium [35]. They can absorb from a minimum of 10 to $20 \%$ to 1000 folds of their dry weight present in water. When a dry hydrogel soaks up the water, the molecules infiltrating the hydrogel matrix moistens the polar hydrophilic groups in it. After the hydration of these oppositely charged groups, the network swells and exposure to the hydrophobic groups occurs [36].

\section{Mechanical Properties}

These properties of the hydrogels can vary relying on the purpose of use of the substance. A gel with high rigidity can be obtained by increasing the cross-linkages in it. In contrast this rigidity can be reduced by heating these materials. Such as gelatin shows an increase in the Young Modulus through cross-linking [37]. The Young Modulus is the result of the interaction between the gel matrix and solution, i.e., water. The hydrogel cross-linking density can be assessed by employing Flory's theory and Young's Modulus [38].

\section{Porosity and Permeation}

The factors that affect the hydrogel matrix include the interconnections between the pores, the average size of the pore and the distribution of pore size. All these factors add up to form a significant parameter called tortuosity [39]. The influencing factors for the distribution of pore-size are as follows:

a) Chemical cross-link concentrations of the polymer strands.

b) Physical entanglements present in the polymer strands.

c) Net charge present on the polyelectrolyte hydrogel.

The influencing factors of the porous structure of a hydrogel include:

a) Type of the surrounding solution.

b) Diffused ionic solutes in solution.

c) Dissolved uncharged solutes.

The high porosity enable them to uptake large amounts of water and swell [40]. The porous microstructure of hydrogels can be determined by various methods [41].

\section{Cross Linking}

The hydrogels are characterized by networks of polymers formed by cross-links. The properties of the hydrogels are altered by the presence of the number of cross-links [42]. The cross-links are of various types and they vary according to the type such as if they are bound physically or chemically [43]. The tailorable characteristics of hydrogels come from the type of cross-linkage and that is how they can be optimized [44]

\section{Methods of Preparation of Hydrogels}

They consist of cross-linked water interacting network of polymer that gives it an elastic structure. To produce a hydrogel such techniques are used that can form a cross-linked polymer [45]. A standard method to produce cross-linkage is the free-radical polymerization. Some ways to cross-link water-soluble linear polymers include:

a) Linking the polymer chains via a chemical reaction

b) Use of ionizing radiation for the generation of main-chain free radicals that can recombine as cross-link junctions

c) Physical interactions, i.e., electrostatics [24] 
As stated in the above classification, they are divided into three classes based on the technique used in their preparation. These are as follows:

\section{Homopolymers}

Cross-linked homopolymers hydrogels are usually used for contact lens production and one way to prepare it is by selecting poly (2-hydroxyethyl methacrylate) as a monomer, cross-linking agent; polyethylene glycol dimethacrylate and UV-sensitive initiator; benzoin isobutyl ether. The cross-linked film is formed in de-ionized water and then treated with UV radiation $(\lambda=253.7 \mathrm{~nm})$ for 20 minutes. The next step is the immersion in water for $24 \mathrm{hrs}$ until fully saturated and non-toxic [46]. Another way to synthesize the poly HEMA hydrogel is the use of low molecular weight crosslinking agent. The use of this agent forms a soft hydrogel containing about 30-40 percent of water and high permeation ability of oxygen. This makes it suitable for contact lenses, soft tissue implants, and carriers for drug delivery [47]. Polyethelene glycol-based hydrogels that are responsive to external stimuli are suitable for efficient and controlled drugs, biomolecules, proteins, and growth factor release. A novel technique of PEG hydrogel formation, introduced by Lin and Anseth, is called as 'Click' chemistry. This method has the advantage of rapid, specific reaction and versatility in bioconjugation [48]. Polyvinyl Alcohol (PVA) hydrogels formation includes interchanging cycles of freezing and thawing. This method of PVA material preparation provides greater mechanical strength as compared to UV radiation. Polyvinyl Pyrrolidone (PVP) hydrogels can be synthesized by radiation technique and used in wound healing [49].

\section{Co-Polymeric Hydrogels}

They comprise of two monomer types from which one is lipophobic (water-loving). Gong et al. produced the triblock poly(ethylene glycol)-poly(ecaprolactone)- poly(ethylene glycol) (PECE) co-polymeric biodegradable hydrogel for drug delivery [50]. The ring-opening copolymerization technique was implied for $\varepsilon$-caprolactone. For triblock synthesis, the initiator used was mPEG, the catalyst was stannous octoate and the coupling agent was hexamethylene diisocyanate. When applied in-situ, this copolymeric block forms a hydrogel. In another study, Kim and his co-workers synthesized copolymers of Methacrylic Acid (MAA) with PEG-PEGDA by using the technique of free-radical photopolymerization. They used tetra (ethylene glycol) dimethacrylate as the cross-linking agent and 1-hydroxycyclohexyl phenyl ketone as an initiator. A nitrogen atmosphere was maintained for 30-minutes, and the process was done under UV light. The hydrogel formed was loaded with insulin successfully [51].

\section{Inter Penetrating Network (IPN)}

IPNs are formed by combining two polymers intimately when one polymer is synthesized in the presence of the other polymer.
This is done by immersion of a pre-polymerized hydrogel in a solution of monomers and an initiator. The main advantages of IPNs are resilient mechanical properties, more efficient drug loading, and controllable physical properties [52]. An example of IPN is the modification of polyethyleneglycol diacrylate hydrogel with $\beta$-chitosan. This modification resulted in improved biocompatibility. This was done by using a 2 percent chitosan solution for mixing a 10 percent aqueous PEGDA solution. UV radiations were used for the formation of cross-links leading to the formation of IPN hydrogel. Kim et al. attempted to extend the applications of another classic biomaterial; Polyurethane (PU), by making its IPN with polyacrylamide (PAA) [53]. The result was an IPN hydrogel that could control water absorption. For this purpose, both PAA and PU were mixed and exposed to UV radiation. The cross-linking agents used for this process were methylenebisacrylamide and vinylpyrrolidone. These types of IPN-PU hydrogels find applications in DDS, artificial muscles, wound dressing material, and sensor systems [54].

\section{Methods of Cross-linking}

Cross-linked networks of natural biopolymers such as alginate, carboxymethylcellulose, and chitosan have been seen. Synthesis polymers such as polyvinyl pyrrolidone [55], polythene glycol [56] , polyacrylic acid [57], polyethylene oxide [58], polymethacrylate [59] and polylactic acid [60] have been cross-linked to form hydrogels. Several methods for the synthesis of hydrogels include physical crosslinking [25], chemical cross-linking [61], grafting polymerization [62], and radiation cross-linking [63]. These modifications can enhance the viscoelasticity and other properties for applications in the pharmaceutical and biomedical field [64].

\section{Physical Cross-linking}

Physical or reversible gels have been a topic of interest because they do not need cross-linking agents for their production and they are relatively easy to produce. Various methods used for crosslinking to produce physical gels include:

Heating or cooling a polymer solution: The hot solutions of carrageenan or gelatin are cooled to form cross-linked gels. The gels formation occurs because of the helix formation and association between the helices [65]. Hennink and Nostrum reviewed the polyethylene glycol-polylactic acid hydrogels formed by physical cross-linking by simply warming the solutions of polymers [25].

Ionic Interactions: This method includes the addition of divalent and trivalent counter ions to cross-link the polymers. Some examples of hydrogels formed by ionic interaction include chitosan-glycerol phosphate salt [66] and chitosan-polylysine [67].

Complex Coacervation: Literature has also shown another method that involves the sticking of oppositely charged polymers and forming complexes that depend on the $\mathrm{pH}$ and concentration of the solutions. Esteban et al. formed a polyionic hydrogel by 
coacervating xanthan and chitosan [68]. Polyionic complexes form as the proteins are positively charged below their isoelectric points and tend to associate with the negatively charged hydrocolloids [69].

Hydrogen Bonding: Hydrogels formed by hydrogen bonding involve reducing the $\mathrm{pH}$ of polymer solutions that have carboxyl groups. Takigami et al. reported the formation of CMC hydrogel by hydrogen bonding after dispersing CMC in a solution of HCL $0.1 \mathrm{M}$ [70].

Freeze Thawing: Freeze-thaw cycling is another way of physically cross-linking the polymers to obtain hydrogels. The principle of this technique is the microcrystal formation after freeze-thawing. Giannouli et al. performed cryogelation of the xanthan polymers to form hydrogel [71].

\section{Chemical Cross-Linking}

It can be done by various techniques that involve the grafting process or linkage of two polymer chains by a cross-linking agent.

Chemical Cross-Linkers: Cross-linking agents such as glutaraldehyde [72] and epichlorohydrin [73] were employed to synthesize hydrogels containing both natural and synthetic polymers. This technique includes the addition of new molecules for producing cross-linked chains in the polymeric chains. Literature also shows the use of 2- acrylamido-2-methylpropanesulfonic acid for cross-linking acrylic acid and $\kappa$-carrageenan for producing biodegradable hydrogels [74]. Carrageenan hydrogels also find applications in the industry for the immobilization of enzymes [75]. Epichlorohydrin can be used as a cross-linker for synthesizing hydrogels from cellulose by heating and freezing techniques [76].

Grafting: Grafting is done by the polymerization of a monomer on a preformed polymer backbone. Grafting can be divided into two types: chemical grafting of radiation grafting. Chemical grafting involves the activation of polymer chains by chemical reagents for example the use of $\mathrm{N}$-vinyl-2-pyrrolidone to graft starch with acrylic acid [77]. Said et al. prepared CMC hydrogel by using electron beam radiation [62].

\section{Radiation Cross-linking}

Another technique for the preparation of these systems is by cross-linking the polymers. This method involves the use of free radical production in the polymer followed by its exposure to a high energy source. It is a useful method as it does not require any chemical additives. It is also a cost-effective process for the modification of biopolymers to be used for biomedical applications [78].

\section{Characterization of Hydrogels}

Morphology, elasticity, and the swelling property are various parameters on which the hydrogels can be characterized. The morphology indicates the structure of the hydrogel or its porosity.
The swelling property indicated the mechanism by which the drug is released from the polymeric material, and the third parameter elasticity shows the strength and stability of the polymeric network and drug carriers respectively [79]. These three parameters are discussed in detail in the following paragraphs.

\section{Morphological Characterization}

The morphology of hydrogels includes its shape, form, and structure and it is determined through a stereomicroscope. The texture of the polymers such as starch, can be assessed by the SEM technique [80].

\section{X-Ray Diffraction}

X-ray diffraction is employed to evaluate the molecular organization and nanoscale structure of an organic hydrogel in its hydrated form. It may also be used to the transition of polymers from their crystalline form to another during the processing [81].

\section{In-Vitro Release Study for Drugs}

The release studies of drugs from the hydrogel carriers are essential to understand the mechanism of release. The duration taken by the hydrogel to release the drug is also of significant importance [82].

\section{FTIR}

The IR absorption spectra of the hydrogels changes if there is any alteration in their morphology. These changes in the spectra can be determined by using FTIR. The appearance of bands shows the cross-linking of the polymers [83]

\section{Swelling Behavior}

The following equation can calculate the swelling percentage (S\%) of the hydrogels:

$$
S(\%)=(W s-W d) / W d * 100,
$$

Wd: Dry hydrogel

Ws: Swollen hydrogel [84].

\section{Rheology}

The viscosity of the hydrogels can be evaluated by the Cone plate viscometer at a constant temperature, i.e., $4^{\circ} \mathrm{C}$ [85].

\section{Measurement of Gel Content}

The gel content can be determined by putting the sample in 200 mesh and washing it three times with distilled water following extraction at $80^{\circ} \mathrm{C}$ in distilled water for $24 \mathrm{~h}$. The remaining gel was dried.

Gel content can be measured gravimetrically as follows:

Gelation $(\%)=\mathrm{Wd} / \mathrm{Wo} * 100$,

Wd: Weight after extraction 
Wo: Initial weight [86].

\section{Applications of Hydrogels}

Hydrogel applications are widespread in various fields, due to their compatibility with different usage conditions and their specific structures. The flexibility of hydrogels makes them easy to be availed in various areas that range from biological to industrial areas. Due to their non- toxic nature and chemical compatibility with biological environments their use extends to medical sciences. Some primary uses of the hydrogels in industry and medicine are as follows:

\section{Drug Delivery}

The astounding characteristics of hydrogels make them a significant candidate for controlled drug delivery systems (systems that deliver the drug at a predetermined rate and time) [87]. This can help to overcome various problems that may occur while handling some formulations. The hydrogels are suitable for the loading and proper release of many drugs because of their high porosity (due to cross-linking and swelling) that, in turn, give them the property of high permeability [88]. The main advantage is that they can be used for sustained release of drugs with a high concentration to a specific area in the body [89]. Studies have also suggested the use of hydrogels for the long term delivery of drugs by gastro-retentive mechanisms [90]. To enhance the binding of a drug to the matrix of hydrogel (to extend the drug release time), both chemical and physical strategies can be used [91]. The drug can be released from hydrogels according to different local changes (stimuli) such as temperature, $\mathrm{pH}$, physical stimuli, or some specific enzymes.

The examples of such hydrogels are as follows:

pH-Sensitive Hydrogels: $\mathrm{pH}$ is one of the most crucial parameters for DDS, as $\mathrm{pH}$ changes occur at many body sites such as the stomach or other specific tissues [92]. To form pH-sensitive hydrogels, both basic and acidic polymers are used, for example:

Acidic Polymers: PAA, Sulfonamide containing polymers [93]

Basic: Ethyl methacrylate, Polyvinyl pyridine [94]

Temperature-sensitive hydrogels in DDS: Temperaturesensitive hydrogels are responsive to changes in the temperature of the body. These can be formed by using thermosensitive polymers, for example, Poly N-isopropylacrylamide and Poly $\mathrm{N}, \mathrm{N}$ diethyl acrylamide [95]. Methylcellulose has also been seen to be triggered by thermal transitions [96].

\section{Dyes and Heavy Metal Ions Removal}

The waste-water of many industrial procedures can cause heavy metal pollution, which can be a severe threat to the health of the public and the eco-systems. Thus, the removal of these dangerous heavy metal ions is of great scientific interest. Hydrogels find applications in this regard as well. They act as adsorbents to remove heavy metals and toxic compounds. The functional groups such as carboxyl, phosphonic, sulfonic, and nitrogen on the surface of hydrogels can favor the absorption of the metal ions [97]. However, the use of hydrogels for heavy metal ion toxicity on large scales is not economical [98]. Studies have reported that hydrogels are excellent dye adsorbents. They can absorb materials with high amounts of methylene blue dye. Polyelectrolytes have been reported to be significant in heavy metal ions removal as they can bind to the oppositely charged metal ions forming complexes [99]. Other examples of hydrogels that can be used for metal ion removals are starch, chitosan, cellulose derivative, and alginate. Other phenomena like chelation and sorption also aid hydrogels in removing metal ions [100].

\section{Biosensors}

A biosensor is a combination of chemical and physical sensors [101]. It is a device used to sense and report a biophysical property of any system. A biosensor has a biological recognition part known as a bio element which makes analyzing biological information possible. Biosensors find applications in the following areas:

a) Point-of-care testing

b) Environmental monitoring

c) Diagnostics [102]

Bio element has different structures similar to enzymes, living cells or tissues and antibodies but the critical aspect is its specificity [103]. The biological molecules can be coupled with sensors by various methods such as covalent bonding, entrapment into membranes or matrix and physical adsorption. Hydrogels have also been manipulated to be used in diagnostic purposes or tests such as ECG (used as medical electrodes). The hydrogels can be used in biosensors by coating them on the sensing device (such as an electrode) or by acting as a 3D matrix or supporting bio elements. Hydrogels can protect the sensor parts in a biosensor by preventing undesirable interactions with cells or biological molecules. Various studies have been performed that depict the potential of hydrogels for cell culture. These can be used in endothelial injury, cardiovascular diseases in which the blood vessels may be reformed to treat the disease, the formation of proteins that can accelerate the growth process and bone remodeling [104].

They can give an excellent environment to enzymes or other biomolecules for the preservation of their activity and functional structure. Hydrogels can also immobilize biosensing elements. Some examples for different biosensors in hydrogel matrices include glucose-responsive hydrogels, DNA, antibody-antigen based sensors and oligonucleotides [105]. Living sensors are another group of biosensors, in which hydrogels are combined with living microorganisms or cells forming living cell-polymer composites [106]. The 3D structures, biocompatibility, and the high water content make the hydrogels suitable for the entrapment for cells 
or bacteria. An example of the living sensor is Arxula adeninivorans LS3 (a biological recognition element) used to determine the biodegradable pollutants in the wastewater [107].

\section{Tissue Engineering}

Tissue engineering refers to the combination of materials, cells, and engineering for the improvement or replacement of biological organs. This requires the searching and finding proper cell types and a suitable scaffold for culturing them in appropriate conditions. Tissue engineering offers the potential for regeneration of almost any tissue or organ in a human body [108]. Hydrogels are an excellent option for a scaffold material due to the similarity of their structures to many tissues. They provide the advantage of minimal invasion for delivery and easy processing in mild conditions [109] The material and scaffold design selection depend upon several variables, such as physical properties, biological properties, and mass transfer properties depending on the environment in which it will be placed and the intended application [110]. For example, the type of scaffold and its structure varies for the production of artificial skin and that for artificial bone. Hydrogels for this purpose can belong to either synthetic or natural materials. It is easy to control the chemistry as well as the structure of synthetic hydrogels, which in turn can help in altering their properties. The natural polymers forming hydrogels have favorable in vivo interaction, for example, chitosan and alginate [111]

In tissue engineering applications, the hydrogels have three purposes, which are:

a) Agents for filling vacant spaces (act as bioadhesives, bulking agents, preventing adhesions)

b) Carriers for bioactive molecules

c) 3D structures for supporting cells

Hydrogel scaffolds based on polymers such as alginate, collagen, and chitosan are generally used as bulking agents [112]. Synthetic hydrogels such as polyethylene glycol act as anti-adhesive materials in conditions such as in the prevention of post-operative adhesions. When acting as vehicles for stabilizing and delivering the bioactive molecules to target tissues, the hydrogels allow the drug delivery to only the desired tissues minimizing toxicity to the other tissues. Some of the examples of their carrier hydrogels include ionically cross-linked alginate hydrogels and glutaraldehyde cross-linked collagen sponges [113]. PVA is another hydrophilic polymer that is finding applications in drug delivery [114]. Hydrogels can act as 3D networks for supporting cells and the formation of an ideal tissue because of being able to be highly hydrated. This makes the hydrogels suitable for the goal of tissue development [115]. Blanchard et al. used the keratin-based hydrogels for cell scaffolds in tissue engineering [116]. Chitosan-based hydrogels such as betaglucan have also been studied as candidates for 2D and 3D scaffolds [117].

\section{Injectable Hydrogel for Regeneration of the Spinal Cord}

SCI defined as a complex degenerative disorder that is caused by growth inhibition due to trauma to the tissues of the spinal cord [118]. The use of hydrogels can sometimes recover these injuries. The viscoelastic hydrogels are converted from a liquid to a gel after being injected into the site of injury. Small spaces or transected parts are formed in SCI, which are filled by hydrogels [119]. These hydrogels can be loaded with therapeutic agents before injecting them into the site of injury. However, the properties of these hydrogel scaffolds should resemble that of spinal cord tissues [120].

The requirements for the designing parameters include:

a) Creating a scaffold for cellular infiltration

b) Maintenance of bioactivity

c) Provision of sustained delivery of loaded agents

d) Tunable and local delivery of therapeutic agents

Design parameters include:

a) Designed scaffold's mesh size,

b) Mechanical characteristics of the gel material

c) Biocompatibility of materials used for injured site

d) Conditions of mild solidification

e) Suitable porosity

f) Rate of degradation

g) Bioactivity

Injectable hydrogels can either be natural or synthetic, having their pros and cons. Examples of some injectable hydrogels include glycidyl methacrylate and polyamidoamine macromers (after undergoing gelation process) [121]. Injectable hydrogels are patient-friendly as they are minimally invasive. They provide an easy mixing of cells or bioactive molecules with the polymer solutions which in turn can quickly form the 3D microenvironments in desired shapes [122]. Enzyme mediated injectable hydrogels such as hydrogels containing tyramine conjugated polymers are used in drug delivery systems and as scaffolds due to their high elasticity [123]. Studies have also been done for the role of hydrogels having antigen-antibody interactions in the formation of an injectable 3D network [124].

\section{Contact Lenses}

Synthetic hydrogels have bio-applications in ophthalmology, especially in contact lenses [125]. The concept of contact lenses was first described in 1508 by Leonardo da Vinci. Polyhydroxyethyl methacrylate lenses were developed in the late 1960s by Professor Otto Wichterle which then started the era of soft lenses [126]. An acceptable contact lens has a high oxygen permeability because 
when a contact lens is placed on the cornea that two main problems that occur include the prevention of oxygen exchange and hypoxic stress (disturbance of natural physiological metabolism of the cornea) [127]. A proper choice of contact lens shape and material is necessary for minimizing these problems [128].

Hydrogels are the best solution for this problem because they can cover the following requirements:
a) Superior mechanical characteristics
b) Oxygen permeability
c) Surface wettability
d) Good optical properties
e) Hydrolysis stability
f) Sterilizing
g) No toxicity
h) Biological tolerance towards living cells [129].

Typical hydrogels used for contact lenses include dihydroxy methacrylates, acrylamides, methacrylic acid and many other monomers [130]. Their right swelling and permeability properties make them suitable and efficient for use in lenses [131].

\section{Colon Specific Drug Delivery}

Hydrogels also find applications in the colon-specific drug delivery. For this purpose, polysaccharides are used as polysaccharidase enzymes are present in high concentration in the colon. These hydrogels provide tissue specificity to the drugs in the colon [132]. The controlled delivery of Ibuprofen has been achieved by hydrogel of guargum with cross-linking agent glutaraldehyde [133]. Dextran based hydrogels are also found to be promising as carriers of therapeutic agents for colon-specific drug delivery [134]

\section{Cosmetology}

Hydrogels are also good candidates for cosmetic use as they impart emulsion stability and conditioning [135]. They are useful as carriers for cosmetic agents in delivery and protection purposes as they liquefy at body temperature [136]. They are also used for aesthetic purposes [137].

\section{Topical Drug Delivery}

Hydrogels are also good candidates for the topical delivery of various therapeutic agents [138]. Hydrogels have been made to deliver a synthetic corticosteroid Desonide which is used as an antiinflammatory. These hydrogels provide moisturizing properties and prevent scaling and dryness [139]. Antifungal formulations like cotrimazole have also been developed as hydrogel formulations [140]. Hydrogels containing extracts of medicinal plants have been synthesized for the treatment of topical dermatitis [141]
Polyacrylic polymers have been found to have promising results in topical drug delivery due to their bioadhesive properties [142].

\section{Modified Dosage Forms}

Biomacromolecules such as heparin can be manufactured as modified dosage forms [143]. Insulin can be delivered to the absorption site with hydrogels by entrapping in a polymer matrix. Cross linkers such as N, N' methylene bisacrylamide have been found to provide maximum entrapment efficiency. Thus, these hydrogels prevent the unwanted degradation of drugs like insulin [144].

\section{Wound Healing}

Hydrogels have been developed to treat cartilage defects by using modified polysaccharide occurring in the cartilage [145]. The aldehyde and methacrylate groups functionalizing the polysaccharides react with the proteins in the skin tissue forming a network where chondrocytes are released [146]. Hydrogels containing honey in a matrix are also being used in wound healing [147]. Cell adhesive hydrogels made of PVA and gelatin in addition to blood coagulants have been shown to ensure better effects [148].

\section{Agricultural Uses}

Hydrogels not only have biomedical applications but also have been used in the agriculture. They can be used to prevent soil erosion as they can hydrate the soil and improve infiltration in soil. Hydrogels are considered as ecofriendly as they can prevent the drying of plants during drought periods. Pesticides have been encapsulated by utilizing hydrogels to improve plant growth and avoid pests. It has also been claimed that hydrogels decrease the leaching of the fertilizers [149].

\section{Food Industry}

Hydrogels are being used in the food industry for various purposes as well. A group of hydrogels called as the bio-based hydrogels are used for packaging various food products. Foods such as vegetables or fruits that can become dry because of loss of water may be packed in bio- based hydrogel packing which will then prevent dehydration and keep them fresh. This biodegradable packaging also helps to prevent the food from contamination by various microbial organisms [150].

\section{Miscellaneous}

Hydrogels also find applications in various products related to hygiene especially in the diaper industry. Super absorbent hydrogels contain such polymers that can provide excellent retention properties that are utilized making the diapers for children. A super absorbent cross- linked polymer starch-g-polyacrylate has been used since 1978 for this purpose in Japan [151]. The diapers and other hygiene products made of SAP hydrogels provide the advantage of moisturized skin, no rashes and improved skin health. 
These hydrogels based diapers also prevent contamination, germ colonization and reduce leakage. The weight of diapers could also be reduced by using these hydrogels other disposable products such as napkins, bed sheets for hospitals and sanitary towels [152].

\section{Limitations of Hydrogels}

In addition to all the merits related to hydrogels, there are some demerits or limitations as well. However, the number of advantages of the hydrogels as carriers for drugs relatively high as compared to the demerits. Most of these limitations can be overcome but some substantial challenges remain to exist with the hydrogels. The main drawback of the hydrogels is that they are expensive. The loading of drug in the hydrogels is a complex process and requires skilled labor and mechanical devices for manufacturing. They are very fragile, so they careful handling. The sterilization of the hydrogels is a complicated process. The concentration of the cross-linkers in the hydrogels is vital as a high concentration may lead to toxicity [153].

\section{Conclusion}

Hydrogels belong to a class of polymeric materials either natural or synthetic; having the ability to entrap large volume of water in their matrix due to their swelling properties and specific and flexible structures. They can be characterized and evaluated by various tests such as their morphology, appearance, viscosity (rheological properties), microscopic techniques for their crystalline structures, their release characteristics for the drugs entrapped in them, the way they accumulate water in them and swell various times their size, the content of gel or the gelation capacity. They have found a wide variety of applications because of their ability to modify the polymeric structures thus helping them to obtain the desired functionality. The areas of hydrogel applications are rapidly expanding day by day. Hydrogels can also be formulated and designed to respond to specific stimuli. These hydrogels are referred to as stimuli-responsive hydrogels. These stimuli-responsive hydrogels can be employed in biosensors such as for the detection of ulcers or other diseases. They are also being employed in tissue regeneration making them good candidates for the treatment of tissue injuries such as spinal cord injury. They have different applications that involve their use in the reduction of environmental waste, including the heavy metals that are usually present in waste-waters, also they are being used in a sanitary product like towels, napkins, and baby diapers. Among their properties, biodegradability and biocompatibility makes them an excellent candidate for biological and industrial applications; for example, they can act as materials for toxic pollutants removal.

\section{References}

1. Rizwan M, Yahya R, Hassan A, Yar M, Azzahari AD, et al. (2017) pH Sensitive Hydrogels in Drug Delivery: Brief History, Properties, Swelling, and Release Mechanism, Material Selection and Applications. Polymers 9(4): 137.

2. Kopecek J (2002) Polymer chemistry: Swell gels. Nature 417(6887): 389-391.
3. Wichterle O, D Lim (1960) Hydrophilic gels for biological use. Nature 185(4706): 117-118.

4. Li Y, Huang G, Zhang X, Li B, Chen Y, et al. (2013) Magnetic hydrogels and their potential biomedical applications. Advanced Functional Materials 23(6): 660-672.

5. Lim F, AM Sun (1980) Microencapsulated islets as bioartificial endocrine pancreas. Science 210(4472): 908-910.

6. Omidian H, JG Rocca, K Park (2005) Advances in superporous hydrogels. Journal of controlled release 102(1): 3-12.

7. Chirani N, Yahia LH, Gritsch L, Motta FL, Chirani S, et al. (2015) History and applications of hydrogels. Journal of biomedical sciences $4: 2$.

8. Das N (2013) Preparation methods and properties of hydrogel: a review. Int J Pharm Pharm Sci 5(3): 112-117.

9. Prestwich GD, DM Marecak, JF Marecek, KP Vercruysse, MR Ziebell (1998) Controlled chemical modification of hyaluronic acid: synthesis, applications, and biodegradation of hydrazide derivatives. Journal of Controlled Release 53(1-3): 93-103.

10. Ahmed EM, Aggor FS, Awad AM, El-Aref AT (2013) An innovative method for preparation of nanometal hydroxide superabsorbent hydrogel. Carbohydrate polymers 91(2): 693-698.

11. Masters KS, Shah DN, Leinwand LA, Anseth KS (2005) Crosslinked hyaluronan scaffolds as a biologically active carrier for valvular interstitial cells. Biomaterials 26(15): 2517-2525.

12. Smetana Jr K (1993) Cell biology of hydrogels. Biomaterials 14(14): 1046-1050.

13. Wallmersperger T, B Kroplin, RW Gulch (2004) Coupled chemoelectro-mechanical formulation for ionic polymer gels-numerical and experimental investigations. Mechanics of Materials 36(5-6): 411-420.

14. Mathur AM, SK Moorjani, AB Scranton (1996) Methods for synthesis of hydrogel networks: A review. Journal of Macromolecular Science, Part C: Polymer Reviews 36(2): 405-430.

15. Angelova N, D Hunkeler (1999) Rationalizing the design of polymeric biomaterials. Trends in biotechnology 17(10): 409-421.

16. Dolbow J, E Fried, H Ji (2004) Chemically induced swelling of hydrogels. Journal of the Mechanics and Physics of Solids 52(1): 51-84.

17. Burkert S, Schmidt T, Gohs U, Dorschner H, Arndt KF (2007) Crosslinking of poly ( $\mathrm{N}$-vinyl pyrrolidone) films by electron beam irradiation. Radiation Physics and Chemistry 76(8-9): 1324-1328.

18. Rosiak JM, F Yoshii (1999) Hydrogels and their medical applications. Nuclear Instruments and Methods in Physics Research Section B: Beam Interactions with Materials and Atoms 151(1-4): 56-64.

19. Brandt KA, SA Goldman, TA Inglin (1988) Hydrogel-forming polymer compositions for use in absorbent structures. Google Patents.

20. Battista OA (1981) Natural protein polymer hydrogels. Google Patents.

21. Roorda W, HE BoddÉ, AG de Boer, HE Junginger (1986) Synthetic hydrogels as drug delivery systems. Pharmaceutisch Weekblad 8(3): 165-189.

22. Tanaka T, Annaka M, Ilmain F, Ishii K, Kokufuta E, et al. (1992) Phase transitions of gels, in Mechanics of swelling. Springer pp. 683-703.

23. Agrawal SK, DeLong NS, Tew GN, Bhatia SR (2008) Structural Characterization of PLA- PEO- PLA Solutions and Hydrogels: Crystalline vs Amorphous PLA Domains. Macromolecules 41(5): 1774-1784.

24. Ahmed EM (2015) Hydrogel: Preparation, characterization, and applications: A review. Journal of advanced research 6(2): 105-121.

25. Hennink WE, CF van Nostrum (2012) Novel crosslinking methods to design hydrogels. Advanced drug delivery reviews 54(1): 13-36.

26. Iizawa T, Taketa H, Maruta M, Ishido T, Gotoh T, et al. (2007) Synthesis of porous poly ( $\mathrm{N}$-isopropylacrylamide) gel beads by sedimentation polymerization and their morphology. Journal of applied polymer science 104(2): 842-850. 
27. Syková E, Jendelová $\mathrm{P}$, Urdzíková L, Lesný $\mathrm{P}, \mathrm{Hejcl} \mathrm{A}$ (2006) Bone marrow stem cells and polymer hydrogels-two strategies for spinal cord injury repair. Cellular and molecular neurobiology 26(7-8): 1111-1127.

28. Wang M, Xu L, Hu H, Zhai M, Peng J, et al. (2007) Radiation synthesis of PVP/CMC hydrogels as wound dressing. Nuclear Instruments and Methods in Physics Research Section B: Beam Interactions with Materials and Atoms 265(1): 385-389.

29. Zhang JT, R Bhat, KD Jandt (2009) Temperature-sensitive PVA/PNIPAAm semi-IPN hydrogels with enhanced responsive properties. Acta Biomaterialia 5(1): 488-497.

30. Lipatov YS (2002) Polymer blends and interpenetrating polymer networks at the interface with solids. Progress in Polymer Science 27(9): 1721-1801.

31. Dhara D, P Chatterji (2000) Swelling and deswelling pathways in nonionic poly (N-isopropylacrylamide) hydrogels in presence of additives. Polymer 41(16): 6133-6143.

32. Bahram M, N Mohseni, M Moghtader (2016) An introduction to hydrogels and some recent applications, in Emerging concepts in analysis and applications of hydrogels. Intech Open.

33. Darnell MC, Sun JY, Mehta M, Johnson C, Arany PR, et al. (2013) Performance and biocompatibility of extremely tough alginate/ polyacrylamide hydrogels. Biomaterials 34(33): 8042-8048.

34.Zohuriaan-Mehr M (2006) Super-absorbents. Iran Polymer Society, Tehran 228: 2-4.

35. Hua F, M Qian (2001) Synthesis of self-crosslinking sodium polyacrylate hydrogel and water-absorbing mechanism. Journal of materials science 36(3): 731-738.

36. Hoffman AS (2012) Hydrogels for biomedical applications. Advanced drug delivery reviews 64: 18-23.

37. Poursamar SA, Lehner AN, Azami M, Barough SE, Samadikuchaksaraei A, et al. (2016) The effects of crosslinkers on physical, mechanical, and cytotoxic properties of gelatin sponge prepared via in-situ gas foaming method as a tissue engineering scaffold. Materials Science and Engineering: C 63: 1-9.

38. Grassi M, Sandolo C, Perin D, Coviello T, Lapasin R, et al. (2009) Structural characterization of calcium alginate matrices by means of mechanical and release tests. Molecules 14(8): 3003-3017.

39. Peppas N, Y Huang, M Torres-Lugo, JH Ward, J Zhang (2000) Physicochemical foundations and structural design of hydrogels in medicine and biology. Annual review of biomedical engineering 2(1): 9-29.

40. Kovacic S, MS Silverstein (2016) Superabsorbent, High Porosity, PAMPSBased Hydrogels through Emulsion Templating. Macromolecular rapid communications 37(22): 1814-1819.

41. Partap S, A Muthutantri, IU Rehman, GR Davis, JA Darr (2007) Preparation and characterisation of controlled porosity alginate hydrogels made via a simultaneous micelle templating and internal gelation process. Journal of materials science 42(10): 3502-3507.

42. Lee KY, Rowley JA, Eiselt P, Moy EM, Bouhadir KH, et al. (2000) Controlling mechanical and swelling properties of alginate hydrogels independently by cross-linker type and cross-linking density. Macromolecules 33(11): 4291-4294

43. Maitra J, VK Shukla (2014) Cross-linking in hydrogels-a review. Am Polym Sci 4(2): 25-31.

44. Okay O (2009) General properties of hydrogels, in Hydrogel sensors and actuators. Springer. p. 1-14.

45. Shantha K, D Harding (2002) Synthesis and evaluation of sucrosecontaining polymeric hydrogels for oral drug delivery. Journal of applied polymer science 84(14): 2597-2604.
46. Tsou TL, Tang ST, Huang YC, Wu JR, Young JJ, et al. (2005) Poly(2hydroxyethyl methacrylate) wound dressing containing ciprofloxacin and its drug release studies. Journal of Materials Science: Materials in Medicine16(2): 95-100.

47. Abdel-Azim AAA, Farahat MS, Atta AM, Fattah AAA (1998) Preparation and properties of two-component hydrogels based on 2-acrylamido-2methylpropane sulphonic acid. Polymers for Advanced Technologies 9(5): 282-289.

48. Lin CC, KS Anseth (2009) PEG Hydrogels for the Controlled Release of Biomolecules in Regenerative Medicine. Pharmaceutical Research 26(3): 631-643.

49. Park KR, YC Nho (2003) Synthesis of PVA/PVP hydrogels having twolayer by radiation and their physical properties. Radiation Physics and Chemistry 67(3-4): 361-365.

50. Gong C, Shi S, Dong PW, Kan B, Gou ML, et al. (2009) Synthesis and characterization of PEG-PCL-PEG thermosensitive hydrogel. International journal of pharmaceutics 365(1-2): 89-99.

51. Kim B, NA Peppas (2003) Poly (ethylene glycol)-containing hydrogels for oral protein delivery applications. Biomedical Microdevices 5(4): 333-341.

52. Qiu Y, K Park (2003) Superporous IPN hydrogels having enhanced mechanical properties. AAPS Pharm Sci Tech 4(4): 406-412.

53. Abraham GA, AA De Queiroz, J San Roman (2001) Hydrophilic hybrid IPNs of segmented polyurethanes and copolymers of vinylpyrrolidone for applications in medicine. Biomaterials 22(14): 1971-1985.

54. Kim SC, D Klempner, KC Frisch, HL Frisch (1977) Polyurethane interpenetrating polymer networks. V. Engineering properties of polyurethane-poly (methyl methacrylate) IPN's. Journal of Applied Polymer Science 21(5): 1289-1295.

55. Razzak MT, D Darwis (2001) Irradiation of polyvinyl alcohol and polyvinyl pyrrolidone blended hydrogel for wound dressing. Radiation Physics and Chemistry 62(1): 107-113.

56. Singh A, M Hosseini, SM Hariprasad (2010) Polyethylene glycol hydrogel polymer sealant for closure of sutureless sclerotomies: a histologic study. American journal of ophthalmology 150(3): 346-351.

57. Onuki Y, Nishikawa M, Morishita M, Takayama K (2008) Development of photocrosslinked polyacrylic acid hydrogel as an adhesive for dermatological patches: Involvement of formulation factors in physical properties and pharmacological effects. International journal of pharmaceutics 349(1-2): 47-52.

58. Khoylou F, F Naimian (2009) Radiation synthesis of superabsorbent polyethylene oxide/tragacanth hydrogel. Radiation Physics and Chemistry 78(3): 195-198.

59. Yang D, Zhang JZ, Fu S, Xue Y, Hu J (2010) Evolution process of polymethacrylate hydrogels investigated by rheological and dynamic light scattering techniques. Colloids and Surfaces A: Physicochemical and Engineering Aspects 353(2-3): 197-203.

60. Palumbo FS, Pitarresi G, Mandracchia D, Tripodo G, Giammona G, et al. (2006) New graft copolymers of hyaluronic acid and polylactic acid: Synthesis and characterization. Carbohydrate Polymers 66(3): 379-385.

61. Barbucci R, G Leone, A Vecchiullo (2004) Novel carboxymethylcellulosebased microporous hydrogels suitable for drug delivery. Journal of Biomaterials Science, Polymer Edition 15(5): 607-619.

62. Said HM, SGA Alla, AWM El-Naggar (2004) Synthesis and characterization of novel gels based on carboxymethyl cellulose/acrylic acid prepared by electron beam irradiation. Reactive and Functional Polymers 61(3): 397-404.

63. Fei B, Wach RA, Mitomo H, Yoshii F, Kume T (2000) Hydrogel of biodegradable cellulose derivatives. I. Radiation-induced crosslinking of CMC. Journal of Applied Polymer Science 78(2): 278-283. 
64. Nho YC, JH Lee (2005) Reduction of postsurgical adhesion formation with hydrogels synthesized by radiation. Nuclear Instruments and Methods in Physics Research Section B: Beam Interactions with Materials and Atoms 236(1-4): 277-282.

65. Funami T, Hiroe M, Noda S, Asai I, Ikeda S, et al. (2007) Influence of molecular structure imaged with atomic force microscopy on the rheological behavior of carrageenan aqueous systems in the presence or absence of cations. Food Hydrocolloids 21(4): 617-629.

66. Zhao QS, Ji QX, Xing K, Li XY, Liu CS, et al. (2009) Preparation and characteristics of novel porous hydrogel films based on chitosan and glycerophosphate. Carbohydrate polymers 76(3): 410-416.

67. Bajpai AK, Shukla SK, Bhanu S, Kankane S (2008) Responsive polymers in controlled drug delivery. Progress in Polymer Science 33(11): 1088 1118.

68. Esteban C, D Severian (2000) Polyionic hydrogels based on xanthan and chitosan for stabilising and controlled release of vitamins. Vol. W00004086 (A1).

69. Magnin D, Lefebvre J, Chornet E, Dumitriu S (2004) Physicochemical and structural characterization of a polyionic matrix of interest in biotechnology, in the pharmaceutical and biomedical fields. Carbohydrate Polymers 55(4): 437-453

70. Takigami M, Amada H, Nagasawa N, Yagi T, Kasahara T, et al. (2007) Preparation and properties of CMC gel. Transactions-Materials Research Society of Japan 32(3): 713-716

71. Giannouli P, E Morris (2003) Cryogelation of xanthan. Food Hydrocolloids 17(4): 495-501.

72. Mansur HS, Sadahira AM, Souza AN, Mansur AAP (2008) FTIR spectroscopy characterization of poly (vinyl alcohol) hydrogel with different hydrolysis degree and chemically crosslinked with glutaraldehyde. Materials Science and Engineering: C 28(4): 539-548.

73. Bo J (1992) Study on PVA hydrogel crosslinked by epichlorohydrin. Journal of applied polymer science 46(5): 783-786.

74. Pourjavadi A, MJ Zohuriaan-Mehr (2002) Modification of carbohydrate polymers via grafting in air. 2. Ceric-initiated graft copolymerization of acrylonitrile onto natural and modified polysaccharides. Starch-Stärke 54(10): 482-488

75. Campo VL, Kawano DF, daSilvaJr DB, Carvalho I (2009) Carrageenans Biological properties, chemical modifications and structural analysis-A review. Carbohydrate polymers 77(2): 167-180.

76. Chang C, Zhang L, Zhou J, Zhang L, Kennedy JF (2010) Structure and properties of hydrogels prepared from cellulose in $\mathrm{NaOH} /$ urea aqueous solutions. Carbohydrate Polymers 82(1): 122-127.

77. Spinelli LS (2008) Adsorption of polymers used in drilling fluids on the inner surfaces of carbon steel pipes. Polymer Engineering \& Science 48(10): 1885-1891.

78. Lugao AB, Malmonge SM (2001) Use of radiation in the production of hydrogels. Nuclear Instruments and Methods in Physics Research Section B: Beam Interactions with Materials and Atoms 185(1-4): 37-42.

79. Khare AR, NA Peppas (1995) Swelling/deswelling of anionic copolymer gels. Biomaterials 16(7): 559-567

80. Szepes A, Blümer C, Mäder K, Kása PJr, Szabó Révész P, et al. (2008) Characterization and drug delivery behaviour of starch-based hydrogels prepared via isostatic ultrahigh pressure. Carbohydrate polymers 72(4): 571-578.

81. Estroff LA (2003) Characterization of an organic hydrogel: a cryotransmission electron microscopy and X-ray diffraction study. Advanced Materials 15(1): 38-42.

82. Yu H, C Xiao (2008) Synthesis and properties of novel hydrogels from oxidized konjac glucomannan crosslinked gelatin for in vitro drug delivery. Carbohydrate polymers 72(3): 479-489.
83. Pal K, A Banthia, Majumdar D (2008) Effect of heat treatment of starch on the properties of the starch hydrogels. Materials letters 62(2): 215218.

84. Yin Y (2008) Study of the swelling dynamics with overshooting effect of hydrogels based on sodium alginate-g-acrylic acid. Carbohydrate Polymers 71(4): 682-689.

85. Schuetz YB, Gurny R, Jordan O (2008) A novel thermoresponsive hydrogel based on chitosan. European Journal of Pharmaceutics and Biopharmaceutics 68(1): 19-25.

86. Sokker H (2009) Synthesis and characterization of hydrogels based on grafted chitosan for the controlled drug release. Carbohydrate polymers 75(2): 222-229.

87. Li J, Mooney DJ (2016) Designing hydrogels for controlled drug delivery. Nature Reviews Materials 1(12): 1-17.

88. Bahram M, Nurallahzadeh N, Mohseni N (2015) pH-sensitive hydrogel for coacervative cloud point extraction and spectrophotometric determination of $\mathrm{Cu}$ (II): optimization by central composite design. Journal of the Iranian Chemical Society 12(10): 1781-1787.

89. Thambi T, Y Li, Lee DS (2017) Injectable hydrogels for sustained release of therapeutic agents. Journal of Controlled Release 267: 57-66.

90. Chen J (2000) Gastric retention properties of superporous hydrogel composites. Journal of controlled release 64(1-3): 39-51.

91. Hoare TR, Kohane DS (2008) Hydrogels in drug delivery: Progress and challenges. Polymer 49(8): 1993-2007.

92. Dong LC, Hoffman AS (1991) A novel approach for preparation of pHsensitive hydrogels for enteric drug delivery. Journal of Controlled Release 15(2): 141-152.

93. Bae YH (2000) pH-sensitive polymer containing sulfonamide and its synthesis method.

94. Emileh AE (2007) Swelling behavior, mechanical properties and network parameters of $\mathrm{pH}$-and temperature-sensitive hydrogels of poly ((2-dimethyl amino) ethyl methacrylate-co-butyl methacrylate) European Polymer Journal 43(5): 1986-1995.

95. Park TG, Hoffman AS (1992) Synthesis and characterization of pH-and/ or temperature-sensitive hydrogels. Journal of Applied Polymer Science 46(4): 659-671.

96. Liang H (2004) Novel method using a temperature-sensitive polymer (methylcellulose) to thermally gel aqueous alginate as a pH-sensitive hydrogel. Biomacromolecules 5(5): 1917-1925.

97. Jing G (2013) Recent progress on study of hybrid hydrogels for water treatment. Colloids and Surfaces A: Physicochemical and Engineering Aspects 416: 86-94

98. Tang Q (2009) Synthesis of polyacrylate/poly (ethylene glycol) hydrogel and its absorption properties for heavy metal ions and dye. Polymer Composites 30(8): 1183-1189.

99. Karadağ E, Hui Sun, Shijun Fan, De Hu, Jianming Lin, et al. (2007) Polyelectrolyte CASA hydrogels for uptake of uranyl ions from aqueous solutions. Journal of applied polymer science 104(1): 200-204

100. Sabaa MW (2012) Crosslinked poly (vinyl alcohol)/carboxymethyl chitosan hydrogels for removal of metal ions and dyestuff from aqueous solutions. Journal of Applied Polymer Science 123(6): 34593469.

101. Han IS (2003) Hydrogel biosensor.

102. Song S, H Xu, Fan C (2006) Potential diagnostic applications of biosensors: current and future directions. International journal of nanomedicine 1(4): 433.

103. Mateescu A (2012) Thin hydrogel films for optical biosensor applications. Membranes 2(1): 40-69. 
104. Kirschner CM, Anseth KS (2013) Hydrogels in healthcare: from static to dynamic material microenvironments. Acta materialia 61(3): 931944.

105. Koetting MC (2015) Stimulus-responsive hydrogels: Theory, modern advances, and applications. Materials Science and Engineering: R: Reports 93: 1-49.

106. Koh W, Revzin A (2002) Poly (ethylene glycol) hydrogel microstructures encapsulating living cells. Langmuir 18(7): 2459-2462.

107. Tag K, Matthias Lehmann, Chiyui Chan, Reinhard Renneberg, Klaus Riedel, et al. (1998) Arxula adeninivorans LS3 as suitable biosensor for measurements of biodegradable substances in salt water. Journal of Chemical Technology \& Biotechnology: International Research in Process, Environmental AND Clean Technology 73(4): 385-388.

108. Dhandayuthapani B (2011)Polymeric scaffolds in tissue engineering application: a review. International journal of polymer science.

109. Lee KY, Mooney DJ (2001) Hydrogels for tissue engineering. Chemical reviews 101(7): 1869-1880.

110. Hollister SJ (2005) Porous scaffold design for tissue engineering. Nature materials 4(7): 518.

111. Drury JL, Mooney DJ (2003) Hydrogels for tissue engineering: scaffold design variables and applications. Biomaterials 24(24): 4337-4351.

112. Zhao X (2001) Synthesis of bioadhesive hydrogels from chitin derivatives. International journal of adhesion and adhesives 21(3): 227-232.

113. Tabata Y, M Miyao, M Ozeki, Y Ikada (2000) Controlled release of vascular endothelial growth factor by use of collagen hydrogels. Journal of Biomaterials Science. Polymer Edition 11(9): 915-930.

114. Cascone M (1995) Evaluation of poly (vinyl alcohol) hydrogels as a component of hybrid artificial tissues. Journal of Materials Science: Materials in Medicine 6(2): 71-75.

115. Tibbitt MW, Anseth KS (2009) Hydrogels as extracellular matrix mimics for 3D cell culture. Biotechnology and bioengineering 103(4): 655-663.

116. Blanchard CR (2002) Keratin-based hydrogel for biomedical applications and method of production.

117. Croisier F, Jérôme C (2013) Chitosan-based biomaterials for tissue engineering. European Polymer Journal 49(4): 780-792.

118. Sekhon LH, Fehlings MG (2001) Epidemiology, demographics, and pathophysiology of acute spinal cord injury. Spine 26(24S): S2-S12.

119. Hejčl A (2008) Biocompatible hydrogels in spinal cord injury repair Physiol Res 57(3): S121-S132.

120. Macaya D, Spector M (2012) Injectable hydrogel materials for spinal cord regeneration: a review. Biomedical materials 7(1): 012001

121. Hacker MC, Nawaz HA (2015) Multi-functional macromers for hydrogel design in biomedical engineering and regenerative medicine. International journal of molecular sciences 16(11): 27677-27706.

122. Perale G (2011) Hydrogels in spinal cord injury repair strategies. ACS chemical neuroscience 2(7): 336-345.

123. Jin R, Pieter J Dijkstra, Clemens A van Blitterswijk, Marcel Karperien, Jan Feijen, et al. (2011) Chondrogenesis in injectable enzymatically crosslinked heparin/dextran hydrogels. Journal of controlled release 152(1): 186-195.

124. Miyata T (1999) Preparation of an antigen-sensitive hydrogel using antigen- antibody bindings. Macromolecules 32(6): 2082-2084.

125. Kirchhof S, Goepferich AM, Brandl FP (2015) Hydrogels in ophthalmic applications. European Journal of Pharmaceutics and Biopharmaceutics 95: 227-238.
126. Michalek J (2010) Hydrogels contact lenses, in Biomedical applications of hydrogels handbook. Springer pp. 303-315.

127. Jones LW, Jones DA (2001) Non-inflammatory corneal complications of contact lens wear. Contact Lens and Anterior Eye 24(2): 73-79.

128. Musgrave CSA, Fang F (2019) Contact lens materials: a materials science perspective. Materials 12(2): p. 261.

129. Xinming L, Sergey V Mikhalovsky, Susan R Sandeman, Carol A Howel, Liao Liewen, et al. (2008) Polymeric hydrogels for novel contact lensbased ophthalmic drug delivery systems: A review. Contact Lens and Anterior Eye 31(2): 57-64.

130. Jeyanthi R, KP Rao (1990) In vivo biocompatibility of collagenpoly (hydroxyethyl methacrylate) hydrogels. Biomaterials 11(4): 238-243.

131. Künzler JF (1996) Silicone hydrogels for contact lens application. Trends in Polymer Science 2(4): 52-59.

132. Singh B, Sharma N, Chauhan N (2007) Synthesis, characterization and swelling studies of $\mathrm{pH}$ responsive psyllium and methacrylamide based hydrogels for the use in colon specific drug delivery. Carbohydrate polymers 69(4): 631-643.

133. Das A, Wadhwa A, A Srivastava (2006) Cross-linked guar gum hydrogel discs for colon-specific delivery of ibuprofen: formulation and in vitro evaluation. Drug delivery 13(2): 139-142.

134. Hovgaard L, Brøndsted H (1995) Dextran hydrogels for colon-specific drug delivery. Journal of Controlled Release 36(1-2): 159-166.

135. Cai W, RB Gupta, Staff UB (2000) Hydrogels. Kirk-Othmer Encyclopedia of Chemical Technology p. 1-20.

136. Gould FE (1991)Thermally reversible polyurethane hydrogels and cosmetic. biological and medical uses.

137. Adams TS, Crook TA, Cadier MA (2007) A late complication following the insertion of hydrogel breast implants. Journal of plastic, reconstructive \& aesthetic surgery 60(2): 210-212.

138. Murphy DJ, Ryan G Loughlin, Ryan F Donnelly, Mark G Jenkins, Paul A M C Carron, et al. (2012) Physical characterisation and component release of poly (vinyl alcohol)-tetrahydroxyborate hydrogels and their applicability as potential topical drug delivery systems. International journal of pharmaceutics 423(2): p. 326-334.

139. Wynne A (2002) An effective, cosmetically acceptable, novel hydrogel emollient for the management of dry skin conditions. Journal of dermatological treatment 13(2): 61-66.

140. Chang JY, Eun Jung Kim, Dong Deuk Jang, Ki Taek Nam, Chong Kook Kim, et al. (2002) Prolonged antifungal effects of clotrimazole-containing mucoadhesive thermosensitive gels on vaginitis. Journal of controlled release 82(1): 39-50.

141. Nho Y (2010) Therapeutic hydrogel for atopic dermatitis and preparation method thereof. Google Patents.

142. Calixto G (2015) Polyacrylic acid polymers hydrogels intended to topical drug delivery: preparation and characterization. Pharmaceutical development and technology 20(4): 490-496.

143. Tae G, Won Il Choi, Mihye Kim, Patrick S Stayton, Allan S Hoffman, et al. (2007) Formation of a novel heparin-based hydrogel in the presence of heparin-binding biomolecules. Biomacromolecules 8(6): 1979-1986.

144. Bajpai S, SS Saggu (2007) Insulin Release Behavior of Poly (methacrylamide-co-N-vinyl-2-pyrrolidone-co-itaconic acid) Hydrogel: An Interesting Probe. Part II. Journal of Macromolecular Science, Part A: Pure and Applied Chemistry 44(2): 153-157.

145. Fenglan X (2004) Preparation and characterization of nanohydroxyapatite/poly (vinyl alcohol) hydrogel biocomposite. Journal of materials science 39(18): 5669-5672. 
146. Baroli B (2007) Hydrogels for tissue engineering and delivery of tissue-inducing substances. Journal of pharmaceutical sciences 96(9): 2197-2223.

147. Yusof N (2007) Development of honey hydrogel dressing for enhanced wound healing. Radiation Physics and Chemistry 76(11-12): 17671770

148. Mukherjee D, Banthia A (2006) Preparation of adrenochrome hydrogel patch, gel, ointment, and the comparison of their blood coagulating and wound healing capability. Materials and manufacturing processes 21(3): 297-301.

149. Rudzinski WE, AM Dave, UH Vaishnav, SG Kumbar , AR Kulkarni, et al. (2002) Hydrogels as controlled release devices in agriculture. Designed monomers and polymers 5(1): 39-65.

\section{ISSN: 2574-1241}

DOI: $10.26717 /$ BJSTR.2021.33.005340

Abdul Qayyum Khan. Biomed J Sci \& Tech Res

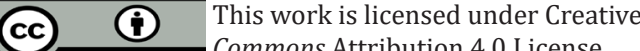

Submission Link: https://biomedres.us/submit-manuscript.php
150. Batista RA, Mayanna Machado Freitasa, Miguel Ângelo Cerqueirad, José António Teixeirae, Juliana Cordeiro Cardosoaf, et al. (2019) Hydrogel as an alternative structure for food packaging systems. Carbohydrate polymers 205: 106-116.

151. Masuda F (1994) Trends in the development of superabsorbent polymers for diapers. ACS Publications.

152. Nizam El Din HM (2012) Surface coating on cotton fabrics of new multilayer formulations based on superabsorbent hydrogels synthesized by gamma radiation designed for diapers. Journal of Applied Polymer Science 125(S2): E180-E186.

153. Kashyap N, Kumar N, M Kumar (2005) Hydrogels for pharmaceutical and biomedical applications. Critical Review in Therapeutic Drug Carrier Systems 22(2): 107.

$\begin{array}{ll}\text { BIOMEDICAL } & \text { Assets of Publishing with us } \\ \text { RESEARCHES } & \text { - Global archiving of articles } \\ \text { - Immediate, unrestricted online access }\end{array}$

